\title{
Les grandes pointes foliacées du type " Ponsonby » : un traceur culturel en Patagonie australe
}

Dominique Legoupil et Nicole Pigeot

\section{(2) OpenEdition \\ Journals}

Édition électronique

URL : https://journals.openedition.org/jsa/10941

DOI : 10.4000/jsa. 10941

ISSN : 1957-7842

Éditeur

Société des américanistes

Édition imprimée

Date de publication : 5 décembre 2009

Pagination : 7-32

ISSN : 0037-9174

Référence électronique

Dominique Legoupil et Nicole Pigeot, «Les grandes pointes foliacées du type «Ponsonby » : un traceur culturel en Patagonie australe », Journal de la Société des américanistes [En ligne], 95-2 | 2009, mis en ligne le 10 décembre 2014, consulté le 03 septembre 2022. URL : http:// journals.openedition.org/jsa/10941 ; DOI : https://doi.org/10.4000/jsa.10941 


\title{
LES GRANDES POINTES FOLIACÉES
}

DU TYPE « PONSONBY ».

\section{UN TRACEUR CULTUREL EN PATAGONIE AUSTRALE}

\author{
Dominique LEGOUPIL et Nicole PIGEOT *
}

De grandes pointes bifaciales foliacées très spectaculaires furent découvertes à Ponsonby en Patagonie australe, par Emperaire, il y a une cinquantaine d'années. La multiplication de ces découvertes dans toute une série de sites (plus d'une dizaine maintenant), nous amène à nous interroger sur la signification et la valeur épistémologique de ces objets. Au-delà de leur fonctionnalité, la mise en évidence de critères diagnostiques personnalisés (chaîne opératoire, automatisme technique, standard morphologique, soin d'exécution, probable valeur sociale...) fait de ces objets les marqueurs incontestables d'un faciès culturel. Ils sont donc des témoins privilégiés qui nous permettent de nous interroger sur leur origine (emprunt ? innovation technique ?) et de suivre l'évolution d'un groupe social représentatif des nomades marins des archipels de Patagonie au $\mathrm{V}^{\mathrm{e}}$ millénaire cal BP. [Mots-clés : pointe bifaciale, marqueur culturel, Indiens canoeros, Ponsonby, Patagonie.]

Las grandes puntas foliáceas tipo de "Ponsonby" ». Un marcador cultural en Patagonia austral. Hace 50 años Emperaire descubrió por primera vez grandes puntas bifaciales foliáceas muy espectaculares en Ponsonby, Patagonia austral. La multiplicación de tales puntas en toda una serie de sitios (actualmente más de una decena), nos lleva a interrogarnos sobre el significado y el valor epistemológico de estos objetos. Más allá de su funcionalidad, la puesta en evidencia de criterios diagnósticos personalizados (cadena operatoria, automatismo técnico, norma morfológica, cuidado de ejecución, probable valor social...) hace de estos vestigios los marcadores innegables de una facies cultural. Son pues los testigos privilegiados que nos permiten preguntarnos sobre su origen (¿préstamo? ¿innovación técnica?) y seguir la evolución de un grupo social representativo de los nómadas marinos de los archipiélagos de Patagonia en el $5^{\circ}$ milenio antes del presente. [Palabras claves : punta bifacial, marcador cultural, indios canoeros, Ponsonby, Patagonia.]

* CNRS, UMR 7041-Ethnologie préhistorique, Maison René-Ginouvès (archéologie et ethnologie), 21 allée de l'université, 92023 Nanterre cedex [dominique.legoupil@mae.u-paris10.fr] ; Institut d'art et d'archéologie, Université Paris 1 Panthéon-Sorbonne, 3 rue Michelet, 75006 Paris [nicole.pigeot@univ-paris1.fr].

Journal de la Société des Américanistes, 2009, 95-2, pp. 7-32. (O Société des Américanistes. 
The large foliaceous points of « Ponsonby » type. A culturalmarker in Southern Patagonia. Large and very spectacular foliaceous bifacial points were discovered in Ponsonby, Southern Patagonia, about fifty years ago by Emperaire. The multiplication of this kind of discoveries in several sites (presently more than ten), calls into question the significance and the epistemological value of these objects. Beyond the question of their function, the description of personalized diagnostic criteria (" chaine opératoire ", technical automatism, morphological standard, very careful execution, social value...) unables us to see these objects as the undeniable markers of a cultural facies. They therefore allow us to speculate about their origin (technical tranfer? technical innovation?) and to track the evolution of a social group representative of the maritime nomads in the archipelagos of Patagonia during the 5th millennium BP. [Key words: bifacial point, cultural marker, canoe Indians, Ponsonby, Patagonia.]

De grandes pointes lithiques bifaciales foliacées, fines et longues, découvertes pour la première fois à Ponsonby, en Patagonie australe, il y a 50 ans, marquent désormais toute une série de sites du $\mathrm{V}^{\mathrm{e}}$ millénaire cal BP dans cette région. Par leur morphologie foliacée (lancéolée) très normalisée, leurs grandes dimensions et une denticulation occasionnelle mais récurrente, elles sont très différentes des autres pointes observées avant, après, ou ailleurs, chez les chasseurscueilleurs, tant terrestres que maritimes, de la région.

La valeur de ces pièces exceptionnelles mérite réflexion. Il est vrai que le façonnage bifacial d'outils ou d'armes est une constante des traditions culturelles observées dans la région et même dans l'ensemble du continent américain à différentes époques de l'Holocène. Toutefois, l'examen de certains aspects techniques et fonctionnels des grandes pointes de Ponsonby indique qu'elles ont acquis le statut d'un véritable marqueur culturel. En considérant leur spécificité conjointement avec leur traçage historique dans le contexte géographique qui leur est spécifique, il nous apparaît que ces pointes peuvent caractériser un groupe original aux traditions communes. On peut même s'interroger sur le rôle social et symbolique qu'elles ont pu jouer, à l'instar d'autres traits culturels.

\section{UN CONTEXTE CHRONOGÉOGRAPHIQUE HOMOGÈNE}

Seuls deux sites à grandes pointes ont fait jusqu'à présent l'objet de fouilles extensives: Ponsonby partiellement fouillé dans les années 1951-1958 par J. et A. Emperaire (Laming-Emperaire 1967-1968 ; Emperaire 1988), puis repris dans une partie restante du site - les locus 1 et 2 - entre 1993 et 1997 (Legoupil 2003a), et Lancha Packewaïa fouillé entre 1973 et 1976 (Orquera et al. 1977 ; Orquera et Piana 1993-1994). Ce dernier a fourni plus d'une centaine de pointes ou fragments de pointes foliacées (Orquera et al. 1977, tabl. IX) ; le premier, 47 pièces (Schidlowsky 2003, tabl. 10). 
Cependant, d'autres pointes similaires (une quinzaine) sont apparues plus occasionnellement dans des sondages ou de petites fouilles limitées, voire lors de collectes de surface dans des sites érodés. On en a ainsi trouvé sur la péninsule de Brunswick à Km 44 (Ortiz-Troncoso 1973) et à Rey Felipe (Miranda 1967), dans la région d'Ultima Esperanza sur l'îlot Krüger et le Rio Hollemberg (Morello et al. 2002), dans le Seno Skyring sur l'île Guzman (Legoupil 1992-1993), enfin sur l'île Dawson dans le Seno Owen (Morello et al. 2002), dans la bahia Fox (Legoupil et al. 2007), ainsi que sur l'îlot Offing où un site, récemment découvert, est en cours de fouille (Legoupil et al. ibid.).

Tous ces gisements se situent dans un cadre chronogéographique bien circonscrit. Ce sont tous des sites côtiers, en prise directe avec la mer, mais situés en lisière du territoire terrestre, le long d'un arc de cercle qui s'étire sur près de $600 \mathrm{~km}$, du Seno Ultima Esperanza au nord-ouest (l'îlot Krüger, Rio Hollemberg), jusqu'au canal Beagle au sud-est (Lancha Packewaïa : voir Figure 1).

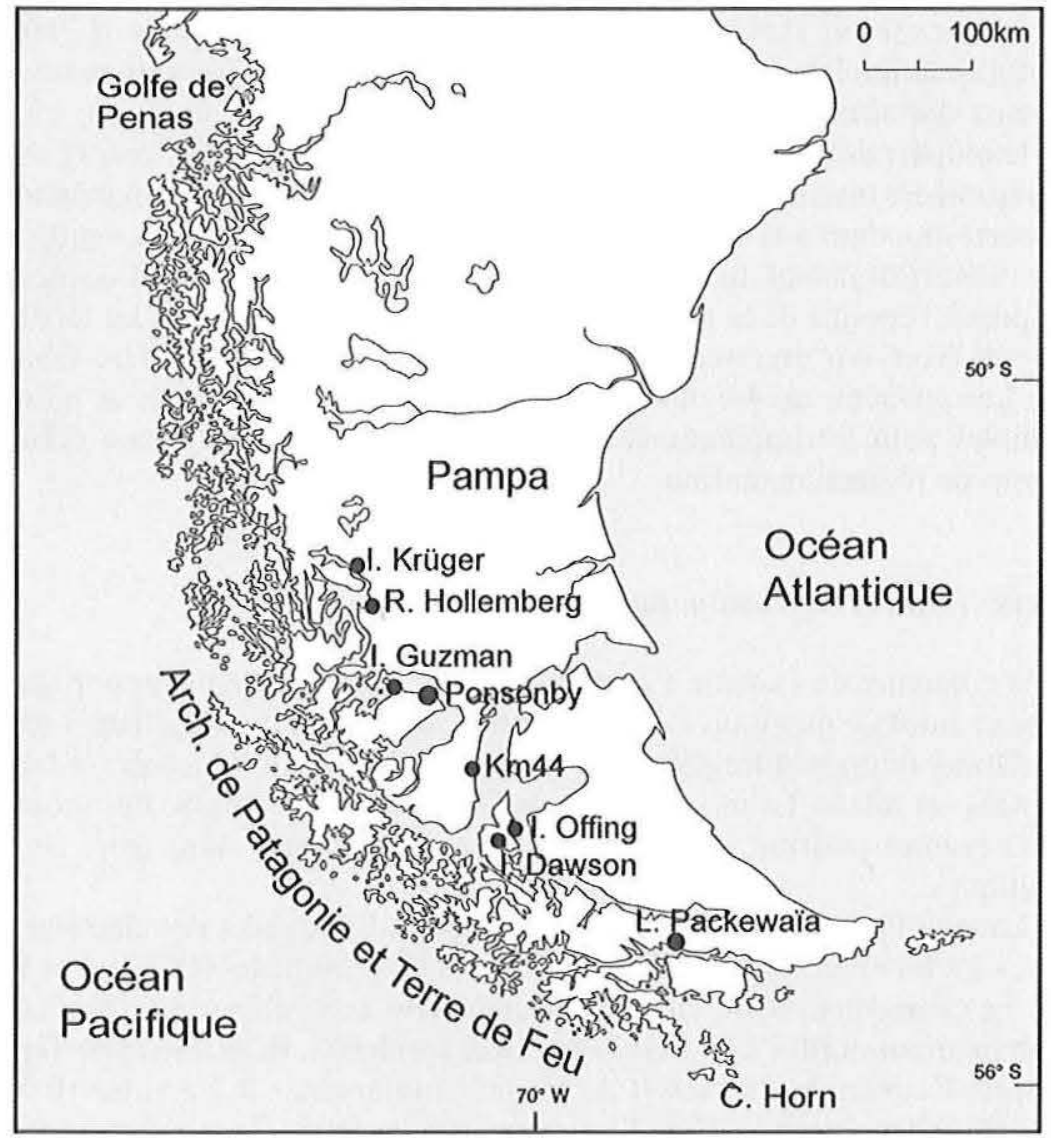

FIG. 1 - Les sites à grandes pointes foliacées : situation géographique. 
La principale plage de recouvrement chronologique des sites varie entre 4220 et 4847 cal BP (Figure 2) ; elle serait donc limitée à une fourchette de moins de 700 ans (Figure 3). Un seul cas pourrait laisser supposer que ces pointes étaient encore fabriquées au début du $\mathrm{III}^{\mathrm{e}}$ millénaire cal BP : celui de $\mathrm{Km} 44$. Toutefois, ce témoignage est fragile : il repose sur l'association d'une pointe découverte il y a 35 ans (Ortiz-Troncoso 1973) et d'un prélèvement daté très récemment dans le site (Morello et al. 2002).

La hauteur des terrasses marines sur lesquelles se trouvent les gisements est étroitement liée à leur période d'occupation. Tous les sites (datés ou non) se trouvent entre 4 et $6 \mathrm{~m}$ au-dessus du niveau actuel des mers ce qui confirme leur grande homogénéité chronologique : $4 \mathrm{~m} 50$ à $5 \mathrm{~m} 50$ à Ponsonby ; environ $6 \mathrm{~m}$ à Lancha Packewaïa (Piana communication personnelle) ; $4 \mathrm{~m}$ à Km 44 (OrtizTroncoso 1973), 6 m à Rio Hollemberg (Morello et al. 2002), 4-5 m à l'île Krüger (Legoupil et al. 2003) ; 4 m 50 à l'île Guzman (Legoupil et al. 1992-1993) ; 4 m à la Bahia Fox sur l'île Dawson, et $5 \mathrm{~m}$ pour l'îlot Offing (Legoupil et al. 2007).

Cette situation intermédiaire entre les terrasses basses $(2-3 \mathrm{~m})$, où se trouvent la plupart des sites tardifs, et les terrasses hautes (au-delà de $10 \mathrm{~m}$ ), où sont situés la plupart des sites anciens (culture d'Englefield et site de Túnel I), est liée à l'évolution des niveaux marins au cours de l'Holocène. Le niveau maximum des eaux, correspondant à la transgression flandrienne, a atteint dans la région entre 5 et $10 \mathrm{~m} \mathrm{snm}$ (au-dessus du niveau marin actuel) vers le milieu de l'Holocène, soit à peu près à l'époque de la première installation de l'homme dans les territoires maritimes (voir, par exemple, la courbe d'Urien et Thurber, in Ortiz-Troncoso 1979). Les terrasses de 4-6 m étaient alors sans doute immergées et n'ont été disponibles pour les populations à grandes pointes qu'après qu'eut débuté le processus de régression marine.

\section{UNE STRATÉGIE ÉCONOMIQUE ORIGINALE}

À la différence du système d'exploitation maritime des premières populations établies en bord de mer dans la partie centrale du détroit de Magellan et dans la mer d'Otway (culture d'Englefield), l'économie observée à Ponsonby et Lancha Packewaïa est mixte. La modification de l'équipement de chasse des groupes à grandes pointes pourrait donc être due à un changement dans leurs activités cynégétiques.

À Lancha Packewaïa, les guanacos, proies traditionnelles des chasseurs terrestres, sont les animaux les plus chassés après les pinnipèdes (Orquera et Piana 1999). Le campement était, en effet, parfaitement accessible à ces artiodactyles qui cohabitèrent durant tout l'Holocène avec les chasseurs terrestres de Terre de Feu, après l'ouverture du détroit de Magellan intervenue il y a entre 10000 et 11000 ans (Clapperton 1992) et l'insularisation du territoire qui s'en est suivie. 


\begin{tabular}{|c|c|c|c|c|c|c|c|c|c|c|c|c|c|c|c|c|c|}
\hline อं & 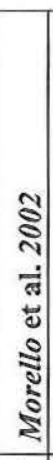 & 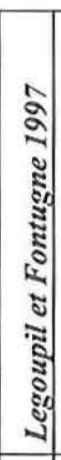 & 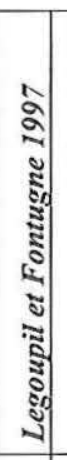 & 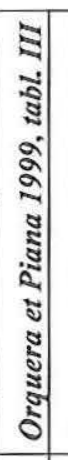 & 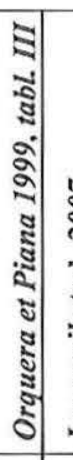 & 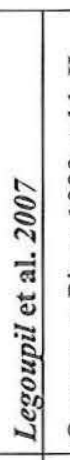 & 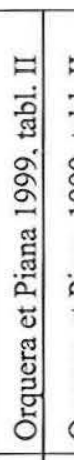 & 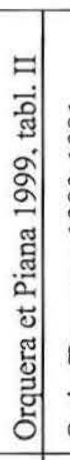 & 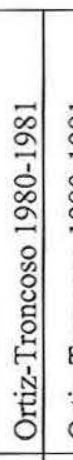 & 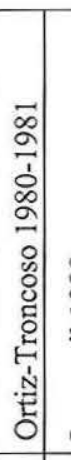 & 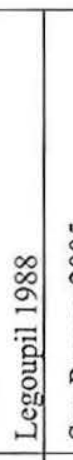 & 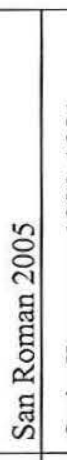 & 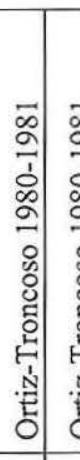 & 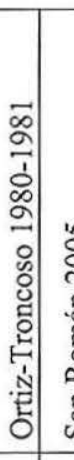 & 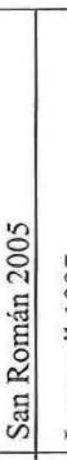 & 월 & 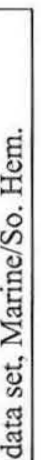 \\
\hline 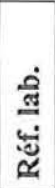 & 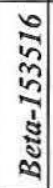 & 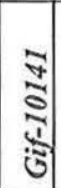 & $\begin{array}{l}0 \\
\frac{1}{5} \\
\frac{7}{5} \\
0\end{array}$ & 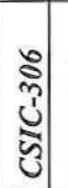 & , & กิ & 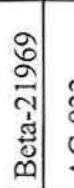 & 今ે & 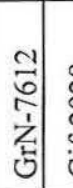 & 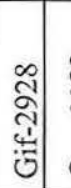 & , & \begin{tabular}{l|}
$\vec{n}$ \\
0 \\
\\
$\vdots$ \\
$\vdots$
\end{tabular} & 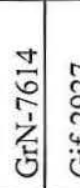 & 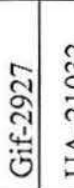 & 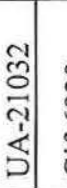 & 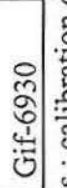 & \\
\hline 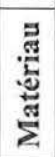 & |ป & 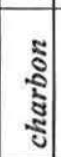 & 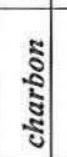 & 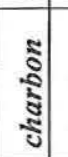 & 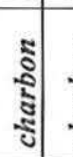 & : & 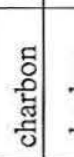 & \begin{tabular}{l|l} 
: \\
: \\
:
\end{tabular} & 总 & 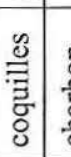 & 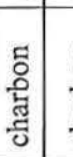 & $\begin{array}{l}\text { 일 } \\
\text { 웡 }\end{array}$ & 든 & 영 & 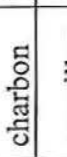 & 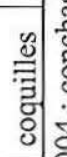 & \\
\hline 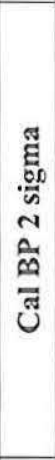 & 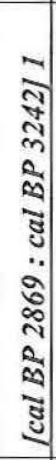 & 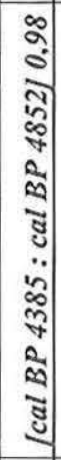 & 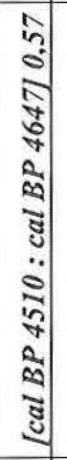 & 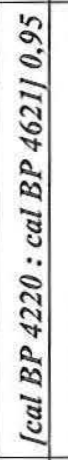 & 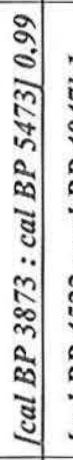 & 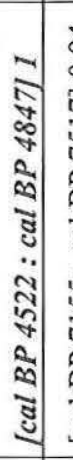 & 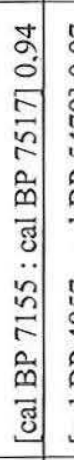 & 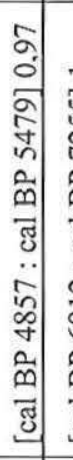 & 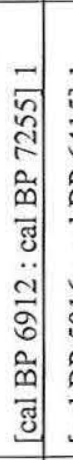 & 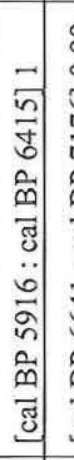 & 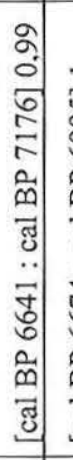 & 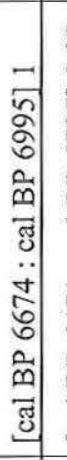 & 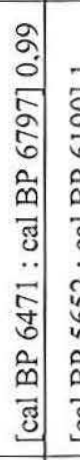 & 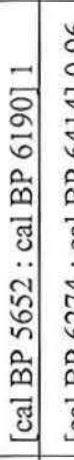 & 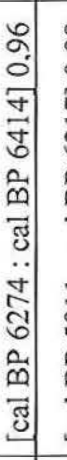 & 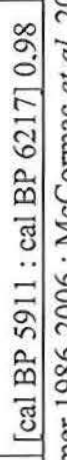 & ¿ \\
\hline$=$ & $\begin{array}{l}8 \\
H \\
8\end{array}$ & $\begin{array}{l}H \\
0 \\
n\end{array}$ & 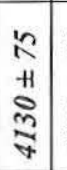 & $\begin{array}{l}R \\
\text { H } \\
\text { ¿ }\end{array}$ & 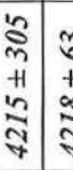 & t & 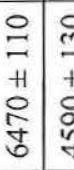 & \begin{tabular}{l|l}
+1 & + \\
8 & 5 \\
5 & 5 \\
8 & 5
\end{tabular} & 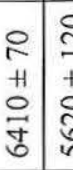 & 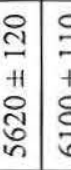 & $\begin{array}{l}+1 \\
8 \\
8 \\
\end{array}$ & \begin{tabular}{l|l}
8 & \\
0 & \\
1 & \\
$n$ & \\
2 & $\vdots$ \\
$\delta$ & $\vdots$
\end{tabular} & 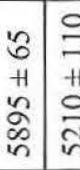 & 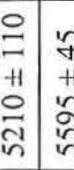 & 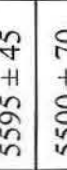 & $\begin{array}{l}8 \\
+1 \\
8 \\
0 \\
n \\
n\end{array}$ & 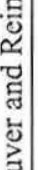 \\
\hline 茕 & 7 & : & & 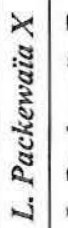 & : & $\sigma^{\prime}$ & 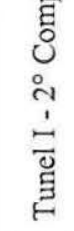 & & 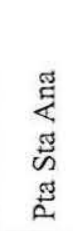 & & $\begin{array}{l}\frac{\partial}{d} \\
\frac{c}{c} \\
\frac{0}{c D} \\
\text { 出 }\end{array}$ & 出 & 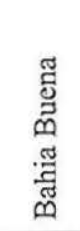 & & $\vec{~}$ & 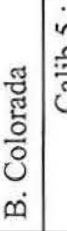 & \\
\hline
\end{tabular}




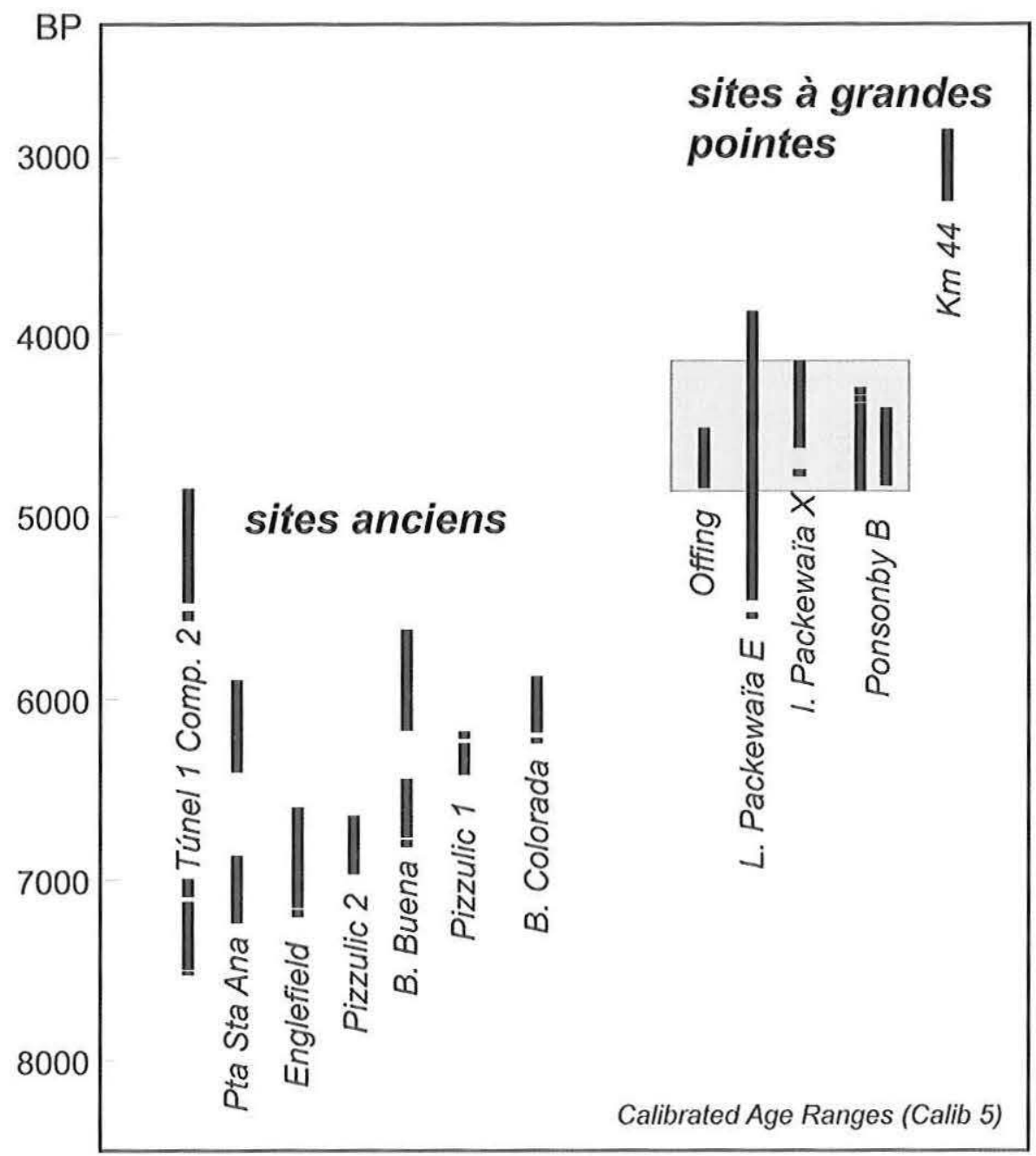

FIG. 3 - Chronologie des principaux sites maritimes anciens et des sites à grandes pointes (en grisé la principale plage de recouvrement des sites).

À Ponsonby, sur l'île Riesco, les guanacos représentaient la majorité des restes retrouvés. L'espèce n'a pas été signalée sur l'île lors de la colonisation à la fin du $\mathrm{XIX}^{\mathrm{e}}$ siècle. Cependant, elle était manifestement présente à l'époque d'occupation du site, quoique la séparation de l'île et du continent ait déjà été effective : celle-ci remonterait au début de l'Holocène, comme l'ouverture du détroit de Magellan, selon Robert D. MacCulloch (communication personnelle). Les guanacos étaient 
même si communs qu'ils se sont fait piéger par dizaines dans une tourbière datée de 7000 ans à Ponsonby (Lepetz et al. 2003). Il y a 4000 à 5000 ans, ils étaient encore chassés massivement par les occupants des niveaux B et C (Lefèvre et al. 2003).

Les données faunistiques manquent pour les autres gisements à grandes pointes, mais tous se trouvent à peu de distance de la pampa et offraient donc un accès facile au territoire steppique ou faiblement boisé des guanacos, notamment les sites de la péninsule de Brunswick ou de la région d'Ultima Esperanza.

En fait, seul le site d'Offĩng ferait exception avec une économie de subsistance qui, d'après les premières analyses, paraît entièrement tournée vers la mer. Seuls quelques os de guanacos, tout à fait anecdotiques, ont été retrouvés dans ce site. Il est vrai que c'est le seul campement à grandes pointes où les occupants n'avaient pas accès directement aux guanacos. En effet, l'îlot Offïng, isolé au milieu du détroit de Magellan, était trop petit (plus ou moins $1 \mathrm{~km}^{2}$ ) pour supporter une population viable de grands mammifères terrestres ; et les guanacos n'ont jamais été signalés non plus sur la grande île Dawson, toute proche, si ce n'est par des vestiges très rares découverts dans des sondages (Legoupil et al. 2007). Les quelques ossements retrouvés à Offïng proviennent donc très probablement, comme ceux-ci, de la Terre de Feu à l'est, ou, éventuellement, de la péninsule de Brunswick à l'ouest. Dans tous les cas, leur présence implique que les occupants de ce campement ont traversé un bras de mer d'une dizaine de kilomètres, infranchissable autrement qu'en canot. Ils représentent donc les seuls possesseurs de grandes pointes dont on sait avec certitude qu'ils pratiquaient la navigation.

Ainsi, les populations à grandes pointes semblent avoir été, sur le plan économique, très adaptables selon les circonstances. Ce caractère mixte les différencie nettement des groupes voisins très spécialisés, généralement observés en Patagonie jusqu'à l'époque moderne. Mais, de ce fait, leur stratégie économique ne représente pas un critère culturel déterminant permettant de les caractériser à coup sûr. Empruntant tantôt aux chasseurs terrestres, tantôt aux chasseurs marins, ils pouvaient pratiquer aussi bien la chasse aux guanacos qu'aux animaux marins ou aux oiseaux. Pratiquant la navigation comme le prouve l'occupation du site d'Offing, ils avaient accès aux deux domaines, terrestre et maritime. Cela les différenciait autant des premiers groupes "canoeros " ${ }^{1}$, installés dans la région et visiblement soucieux de se maintenir à l'écart des chasseurs terrestres grâce à la barrière de l'eau, que des derniers chasseurs terrestres dont on sait qu'ils ne pratiquaient pas la navigation.

\section{UN ÉQUIPEMENT TECHNIQUE À LA CONFLUENCE ENTRE CHASSEURS TERRESTRES ET MARITIMES}

Si l'on examine maintenant l'équipement technique de ces groupes, les principales données dont on dispose proviennent, encore une fois, essentiellement de 
Lancha Packewaïa (Orquera et al. 1977; Orquera et Piana 1999; Álvarez 2007) et Ponsonby (Legoupil 2003b, c; Pigeot 2003; Schidlowsky 2001, 2003, 2006). La panoplie, constituée d'armes, d'outils et d'objets décoratifs, présente des caractères communs aux deux groupes socioculturels de la pampa et des archipels.

L'industrie osseuse est typique des populations maritimes: des pointes de harpons monodentées, des pointes de lances multidentées et des outils à extrémité biseautée en os de mammifères marins, ainsi que des poinçons, des tubes et des perles tubulaires sur os d'oiseaux. Elle présente cependant quelques points originaux par rapport à l'industrie des premiers groupes canoeros. Ainsi les pointes de harpons sont plus rares, et techniquement et typologiquement moins systématisées. Leur décoration est aussi beaucoup moins développée que sur les harpons de la culture d'Englefield, caractérisés par des gravures géométriques (Emperaire et Laming 1961 ; Ortiz-Troncoso 1979 ; Legoupil 1997), et ceux des sites anciens du canal Beagle, ornés de décors gravés, à motifs géométriques et naturalistes comme les harpons vulpicéphales du second niveau de Túnel I (Orquera et Piana 1986-1987). Surtout, on a découvert à Ponsonby des fragments de grandes pièces interprétées comme pointes (de sagaies ?) en os de mammifères marins, massives et de section circulaire, uniques dans la région : elles pouvaient atteindre une trentaine de centimètres de longueur pour un diamètre de 2 à $3 \mathrm{~cm}$ et étaient, pour certaines, marquées à une extrémité par deux petits reliefs latéraux courts et épais, plus fonctionnels comme système de fixation dans une hampe que comme barbelure pour retenir une proie (Figure 4, gauche). Des pièces très comparables, mais avec un seul relief, étaient présentées en 1981 dans les vitrines du Museo del Fin del Mundo à Ushuaïa (Figure 4, droite) : elles provenaient de ramassages de surface dans un site du canal Beagle, Rio Chico 3, malheureusement non daté.

Outre les grandes pointes, l'industrie lithique est, elle, essentiellement constituée de grattoirs, de racloirs, de couteaux, de percuteurs et d'éclats utilisés. Toutes ces pièces se retrouvent en proportion variable, selon les activités développées dans les sites, et ne sont pas culturellement très diagnostiques, sauf dans quelques cas comme les grands racloirs à retouches couvrantes, les petits grattoirs unguiformes, ou les bolas. Les grands racloirs présents à Lancha Packewaïa et à Ponsonby évoquent fortement ceux de la grotte Fell (Schidlowsky 2003). Les petits grattoirs (il en existe un second type d'un module plus grand), bien connus chez les chasseurs terrestres, sont représentés à Ponsonby, mais absents à Lancha Packewaïa, ce que Schidlowsky (ibid., p. 200) explique par le fait que « les contacts avec les chasseurs terrestres sont favorisés par la position géographique du gisement de Ponsonby ». Quant aux bolas, armes caractéristiques des chasseurs terrestres, elles ont été façonnées sur place à Ponsonby comme en attestent plusieurs fragments d'ébauches cassées (Legoupil 2003b). Elles ne résultent donc pas d'un simple échange 


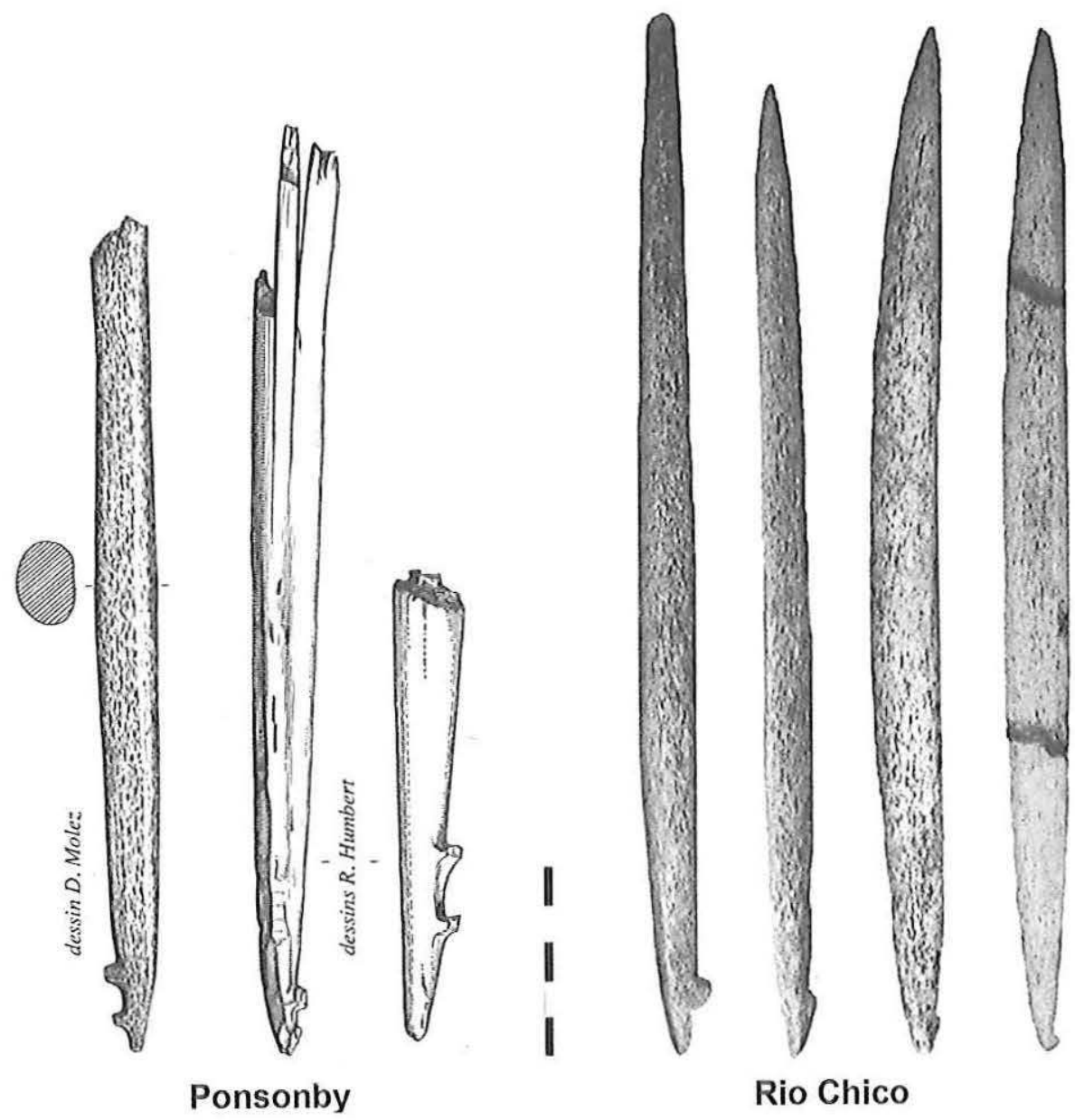

Fig. 4 - Pointes de sagaies de Ponsonby (d'après Legoupil 2003c, figure 5) et Rio Chico 3 (cliché Legoupil).

d'objet, mais d'un véritable emprunt technique intégré jusque dans ses procédés de fabrication...

En fait, c'est surtout dans le choix des matières lithiques qu'on observe l'originalité des sites à grandes pointes (Schidlowsky 2006). Ainsi, à Ponsonby comme à Lancha Packewaïa, sont employés systématiquement deux types de roches, l'une relativement tendre et friable, l'autre plus siliceuse et dure.

Dans le premier site, l'une, appelée communément « lutite », est en réalité une cinérite retravaillée résultant de l'accumulation mécanique de détritus pyroclas- 
tiques et/ou volcaniques plus anciens ; l'autre, la rhyolithe, est une roche pyroclastique primaire résultant d'accumulation directe de matériaux clastiques engendrés par des explosions volcaniques (Terradas communication personnelle, 2003). Dans le second site, il s'agit d'une vulcanite (l'andésite) et de métamorfites (rhyolithes et cinérites), roches pyroclastiques primaires de la Formation Lemaire (selon Terradas et al. 1991). Dans les deux sites, les pointes sont réalisées préférentiellement (mais non exclusivement) sur la roche la plus tendre, alors que la roche plus dure et clastique, au grain plus fin et aux arêtes plus coupantes, était préférée pour le débitage d'éclats (Orquera et Piana 1999 ; Pigeot 2003 ; Álvarez 2007). Dans tous les cas, ces matériaux se distinguent nettement de l'obsidienne utilisée massivement dans les sites d'Englefield (Pigeot et Schidlowsky 1997) et à laquelle les groupes à grandes pointes ne semblent pas avoir eu accès. Ils se différencient également des roches utilisées dans la pampa, en particulier dans la grotte Fell où domine le basalte, suivi de la calcédoine et d'autres roches siliceuses (Schidlowsky 2006, figure 4).

On voit ainsi se dessiner peu à peu les caractères particuliers des sites à grandes pointes : à leur situation chronogéographique limitrophe entre steppe et archipels (mais toujours côtière) vient s'ajouter la polyvalence de leur économie et de leur équipement technique, qui évoque tantôt les groupes maritimes, tantôt les groupes terrestres.

\section{UN MARQUEUR CULTUREL FORT : LES GRANDES POINTES FOLIACÉES}

La caractéristique la plus frappante de ces sites reste les grandes pointes foliacées ou subfoliacées. Il convient donc de s'interroger sur la charge culturelle de ces artefacts : leurs caractères particuliers sont suffisamment diagnostiques et répétés pour que ces pièces constituent véritablement le marqueur d'un groupe socioculturel original. En d'autres termes, on peut considérer qu'elles représentent un type, "le type Ponsonby », au même titre que les pointes Clovis, les pointes « en queue de poisson » ou les pointes de Paiján.

\section{Les critères morphotypologiques}

Deux critères permettent de distinguer facilement ces pointes : leurs dimensions exceptionnelles et le modèle morphologique très standardisé vers lequel elles tendent (Figure 5).

La longueur des pièces de Lancha Packewaïa varie en général entre 12 et $17 \mathrm{~cm}$ (Orquera et Piana 1999), celles de Ponsonby entre 13 et $16 \mathrm{~cm}$ (Schidlowsky 2003). Presque toutes les pièces entières provenant des autres sites entrent peu ou prou dans ce cadre (Morello et al. 2002, tabl. 1). Toutefois, quelques pièces exceptionnelles sortent du lot : un peu plus grandes (jusqu’à 


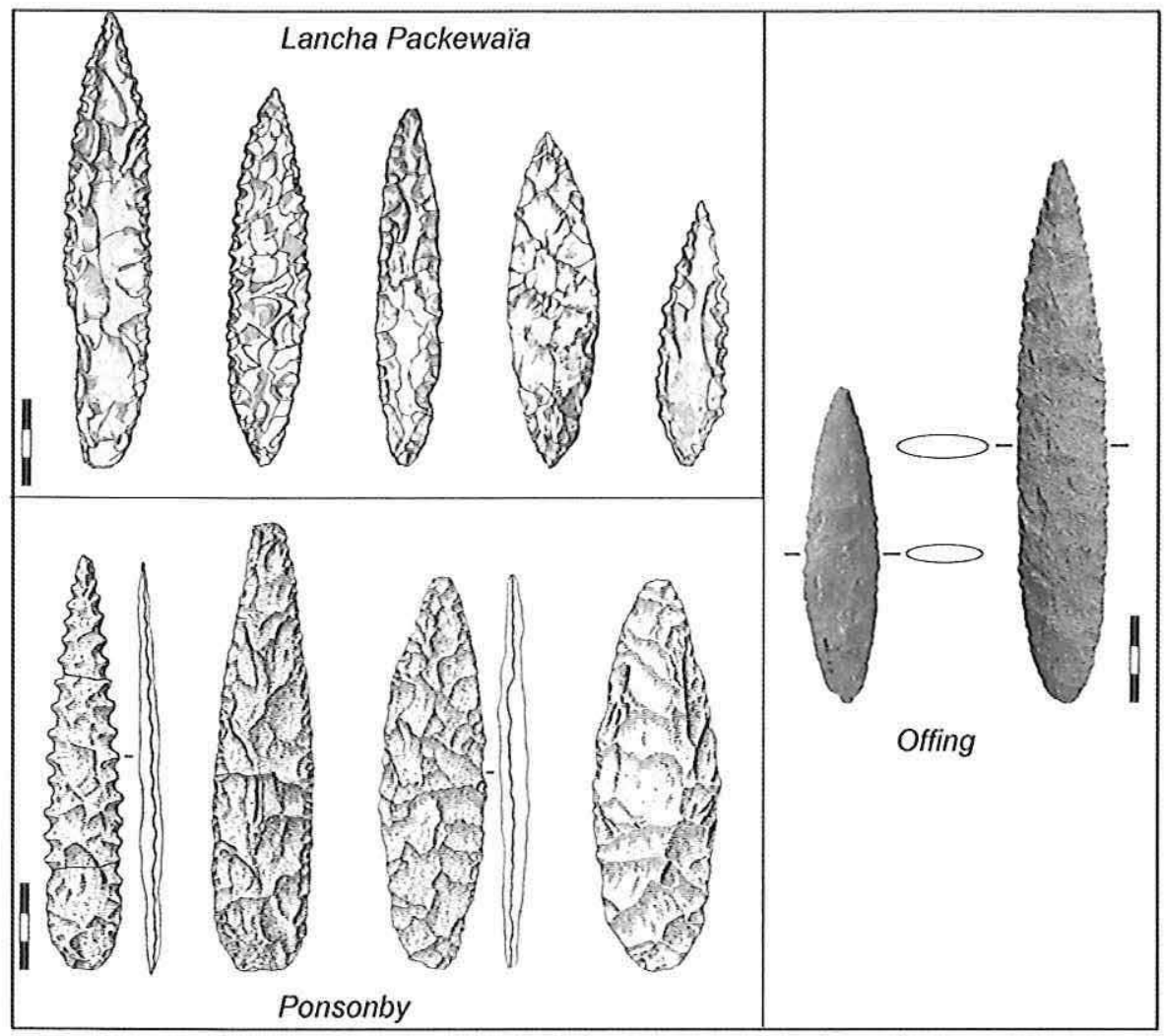

FIg. 5 - Les grandes pointes foliacées de Lancha Pachewaïa (Orquera et al. 1977, figures 48, 46, 45, 43, 49), de Ponsonby et d'Offing.

$20 \mathrm{~cm}$, par exemple, à Ponsonby et à Offing) ou un peu plus petites (une dizaine de centimètres à Lancha Packewaïa et à Offĭng).

La longueur moyenne s'établit ainsi autour d'une quinzaine de centimètres. C'est le double, voire le triple, de celle des pointes observées au cours de la première moitié de l'Holocène en Patagonie, aussi bien dans les sites anciens de l'île d'Englefield, notamment à Bahia Colorada (Pigeot et Schidlowsky 1997, figure 30), que dans les couches moyennes (III et IV) de la grotte Fell (Bird 1993, figures 14 et 15 ).

Morphologiquement, les fouilles du locus 1 de Ponsonby n'ont pas permis de mettre en évidence l'existence de types ou de sous-types standardisés (Pigeot 2003). En revanche, en incluant les pièces de la collection Emperaire, Schidlowsky (2001) a distingué deux grandes catégories de pièces bifaciales : quelques bifaces cordiformes (de longueur similaire aux pointes, mais plus larges et plus épais) et 
les pointes elles-mêmes, très largement majoritaires. Ce sont ces dernières qui représentent le type " classique ", foliacé : allongé, mince, symétrique, appointé à une extrémité, plutôt arrondi à l'autre et parfois denticulé. Outre leur longueur absolue, l'allongement de ces pièces est particulièrement spectaculaire, notamment par rapport aux bifaces cordiformes de Ponsonby ou aux nombreuses ébauches de Lancha Packewaïa qui auraient pu être destinées aussi bien au façonnage de pointes que de simples bifaces. Cet allongement des pointes (rapport longueur/largeur) est généralement compris entre 3,3 et 3,9 à Ponsonby (Schidlowsky 2003, p. 188), mais il peut atteindre un rapport de 5 et même 6, par exemple sur une des pointes de l'île Offing (Figure 5, à droite). Ces pointes sont également assez minces malgré leurs grandes dimensions : leur épaisseur varie entre 11 et $17 \mathrm{~mm}$ à Ponsonby (Schidlowsky 2003, tabl. 9), 8 et $12 \mathrm{~mm}$ sur l'île Kruger et les sites de la péninsule de Brunswick (Morello et al. 2002, tabl. 1) et moins de $10 \mathrm{~mm}$ pour les pièces représentées dans Orquera et al. (1977). Selon ces auteurs, le rapport épaisseur/largeur est alors de 1/4.

La recherche d'une longueur maximale de tranchant avait déjà été observée dans les sites d'Englefield, mais pour les couteaux en obsidienne (Pigeot et Schidlowsky 1997), ce qui était fonctionnellement justifié. Dans le cas des pointes, cette tendance ne s'expliquerait que si elles étaient utilisées comme couteau mais, dans ce cas, il n'y aurait aucune raison de se soucier d'une parfaite symétrie. Pour une utilisation en pointe de jet ou de poignard, la longueur et, surtout, l'allongement fragilisent considérablement la pièce sans, pour autant, offrir une plus grande force de pénétration. C'est ce choix qui explique sans doute le grand nombre de pièces fragmentaires retrouvées dans les sites, qu'elles aient été cassées en deux lors de leur fabrication (comme c'est le cas le plus fréquent), ou en percussion comme l'évoquent certaines fractures en biais portant une languette.

\section{Le système technique et la chaîne opératoire de façonnage des pointes}

Le façonnage bifacial est pratiqué en Patagonie depuis la fin du Pléistocène et les débuts de l'Holocène, comme en témoignent les pointes en queue de poisson caractéristiques notamment de la phase I de la grotte Fell (Bird 1993). Il a toutefois connu un développement apparemment à éclipses selon les moments, les régions et les sites, dans bien des cas, sans doute, pour des raisons fonctionnelles (selon les activités auxquelles elles étaient destinées) et non en fonction de variations culturelles. Peut-être est-ce dû aussi au hasard du développement des recherches. Ainsi, un millénaire avant l'apparition des grandes pointes, la taille bifaciale était utilisée massivement pour réaliser toutes sortes d'objets, outils ou armes dans l'ensemble des sites à obsidienne de la culture d'Englefield. À la même époque, elle était à peine représentée dans le second niveau de Tunel I, où aucune pointe n'était observée, sauf une, en obsidienne, d'origine allochtone (Orquera et al. 1977). 
Les méthodes employées pour la fabrication des grandes pointes ne constituent pas en elles-mêmes une innovation particulière : le processus de réduction est classique, d'un ébauchage plus ou moins poussé sur masse ou éclat à la percussion directe, surtout tendre, jusqu'à, dans certains cas, une ultime finition à la pression. Mais ce qui fait la marque de fabrique de ce façonnage est sans aucun doute la systématisation de la chaîne opératoire, la qualité et la régularité des réalisations (on pourrait presque parler d'automatisation vu l'homogénéité des produits obtenus malgré les difficultés techniques posées par la dimension des supports), ainsi que le soin apporté à un aménagement final par pression, destiné à produire une denticulation du tranchant qui peut, dans certains cas, être très prononcée (Figure 6).

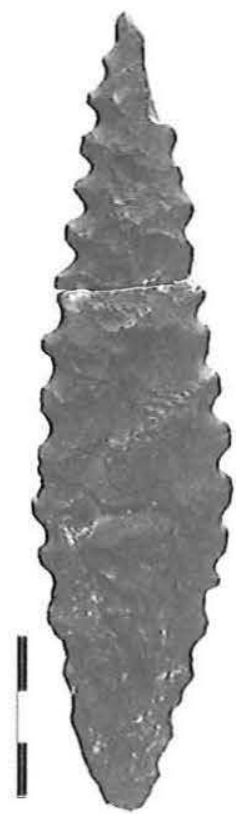

FIG. 6-Pointe denticulée découverte par un pêcheur dans le Seno Owen sur l'ile Dawson (cliché Legoupil).

La chaîne opératoire ${ }^{2}$ de façonnage de ces pièces a été analysée sur toute sa séquence dans le locus 1 de Ponsonby (Pigeot 2003), et c'est surtout à partir de cette étude que nous allons la synthétiser. Le processus mis en œuvre a ainsi été bien identifié depuis l'acquisition des matières et le choix des supports jusqu'à l'abandon des pièces. Il présente des particularités qui sont propres à cet ensemble de pièces et qui se différencient clairement du mode d'acquisition des groupes 
canoeros anciens ou tardifs puisque l'obsidienne réapparaît ensuite dans les sites après un ou deux millénaires d'absence.

\section{L'acquisition des supports}

Comme on l'a vu pour l'ensemble du matériel lithique, les comportements liés aux choix des matières ont été comparables dans les deux sites de référence, pourtant distants de plus de $500 \mathrm{~km}$. Dans le locus 1 de Ponsonby les produits de façonnage sont, à $62 \%$, sur lutite et, à $26 \%$, sur rhyolithe (Pigeot 2003, tabl. 2). À Lancha Packewaïa, la matière la plus utilisée est la vulcanite, suivie par les métamorfites (Orquera et Piana 1999). Le choix préférentiel des roches tendres est manifeste dans les deux cas. À Lancha Packewaïa, le choix de la vulcanite serait justifié par sa bonne homogénéité. À Ponsonby, celui de la lutite serait dû avant tout à la grande dimension des rognons, principale qualité recherchée selon Pigeot (2003), mais aussi à la « finesse du grain, la structure homogène, et la bonne aptitude à la taille » de cette matière, malgré une qualité de tranchant plus médiocre que la rhyolithe (ibid. p. 128).

Si le choix des qualités de matières est identique, celui des supports est, lui, étonnamment différent entre les deux sites : dans un cas des éclats, dans l'autre des masses centrales. À Ponsonby, dans tous les cas déterminables (5 ébauches), les pointes étaient façonnées à partir de grands éclats détachés au moyen d'une forte percussion dure, tandis qu'il n'existe aucune trace d'un façonnage sur galet ou plaquette. La seule pièce qui pourrait représenter une ébauche sur masse est ambiguë et a plutôt été interprétée comme un nucléus relevant de la famille conceptuelle des discoïdes (ibid., figure 6). L'obtention des grands éclats dévolus au façonnage des grandes pointes a donc nécessité la mise en œuvre d'opérations de débitage particulières, car il n'y a pas de nucléus aussi grands dans le niveau d'occupation. Avant d'être rapportés au campement, les grands éclats-supports avaient dû être débités directement sur les plages voisines où abondent les roches transportées par les glaciers. Il s'agit d'une pratique d'acquisition simple et efficace consistant à percuter sur place de gros blocs, sans même avoir à les déplacer, et à obtenir facilement les grands éclats dont la forme est, dès l'origine, assez idéale pour initier un façonnage grâce à la structure déjà bifaciale d'un éclat brut.

À Lancha Packewaïa, en revanche, la première phase de mise en forme est dominée par des témoins spectaculaires: les grandes préformes sur masse centrale (Orquera et al. 1977). Certaines pointes pourraient avoir été réalisées sur éclats, mais aucun témoin de ce procédé n'est signalé, peut-être en raison de la difficulté à identifier les supports initiaux sur des objets entièrement façonnés. Les grandes préformes sur masse attestent en tout cas que des blocs volumineux ont été rapportés au campement, vraisemblablement après avoir été ébauchés sur leur lieu de collecte. Étant donné le poids des pièces, cette collecte était plus coûteuse en énergie qu'à Ponsonby. Cela est d'autant plus vrai que la source de la 
vulcanite n'a pu être retrouvée et pourrait se situer géologiquement hors du canal Beagle (Orquera et Piana 1999), à moins que des rognons n'aient été apportés par le déplacement des glaciers.

L'acquisition des supports lithiques à Ponsonby (et peut-être à Lancha Packewaïa) est en tout cas très différente de ce qui se pratiquait pour l'obsidienne, principale matière utilisée dans les sites canoeros anciens de la culture d'Englefield. Dans ces derniers sites, ce matériau était utilisé indifféremment pour le débitage d'éclats et le façonnage de pièces bifaciales. Surtout, la collecte de ce matériau rare impliquait la connaissance de l'une de ses sources, sans doute dans la zone sud du Seno Otway (Morello et al. 2004 ; Stern et Prieto 1991), et un déplacement par mer (donc une programmation). Les blocs de matière première (sous forme de galets, de baguettes ou, souvent, de plaquettes allongées favorisant l'obtention de produits allongés, notamment des couteaux) étaient rapportés et stockés au campement où de nombreux nodules bruts ou à peine testés ont été retrouvés, en particulier à Bahia Colorada (Pigeot et Schidlowsky 1997).

\section{Le façonnage}

À Ponsonby, le façonnage pouvait commencer directement à la percussion tendre sur ces morphologies déjà « prêtes » que sont les éclats. Il n'y a donc pas de phase d'ébauchage proprement dite. Plus d'un millier d'éclats, courbes et aux talons déversés, très caractéristiques de cette technique, ont été reconnus comme déchets de façonnage à la percussion tendre organique. Ces vestiges témoignent de la fabrication des grandes pointes in situ, une partie d'entre elles étant laissée sur place (au total 27 pointes entières ou fragmentaires après raccords), l'autre emportée ailleurs comme en témoignent certains ensembles auxquels seul manque l'objet final, la pointe elle-même (17 pointes "fantômes » ont ainsi été emportées). Des remontages ont été testés et réalisés, mais avec moins d'insistance pour le façonnage que le débitage, du fait du moindre intérêt technologique des reconstitutions de pointe. En fait, c'est le rapprochement des variétés de roche au niveau très spécifique de chaque matériau taillé qui s'est révélé un excellent substitut au remontage. Cette méthode d'étude a permis de reconnaître, à côté des 27 pointes, le matériel " positif » en quelque sorte, un matériel "négatif » de 17 pointes façonnées sur place avant d'être emportées.

Le schéma de façonnage consiste à utiliser la structure bifaciale pré-existante et à amincir le support de proche en proche, en contrôlant la silhouette et la régularité des bords et des surfaces, jusqu'à l'obtention de la forme souhaitée : mince, allongée, appointée, symétrique. La chaîne est donc continue, réalisée de bout en bout au percuteur tendre organique sur des éclats-supports dont la forme favorable est préalablement déterminée. Cette option offre l'avantage d'une grande facilité tactique par rapport au processus de façonnage classique, sur bloc. Ce dernier oblige à une première phase de réduction effectuée au percuteur dur, ce qui crée des négatifs très « sécants » impropres à l'amincissement postérieur de la 
pointe ; pour affiner la pièce, il faut alors changer d'outil et passer au percuteur tendre. À Ponsonby, le choix d'un façonnage direct au percuteur organique permet d'éviter ce moment de transition toujours délicat, qui ne doit intervenir ni trop tôt, ni surtout trop tard (Pigeot 2003, p. 151).

Enfin, une ultime opération, partielle et peu envahissante, pouvait être effectuée le long des bords par une technique par pression, provoquant la formation d'une denticulation plus ou moins forte qui a pu améliorer les qualités tranchantes de la pièce. Parfois, cette finition est nettement marquée, laissant supposer que c'était bien la forme dentelée des bords qui était visée, et non la régularisation des surfaces actives. Cette denticulation n'était cependant pas systématique (la seule minceur de l'objet pouvant suffire à son efficacité) et l'on peut s'interroger sur le statut des pointes non denticulées et se demander si elles peuvent être considérées comme parfaitement terminées.

La plupart des vestiges de façonnage ont été retrouvés dans deux ateliers spécialisés situés, l'un dans la couche B1, l'autre dans la couche B2 (Legoupil 2003d, figures 35,41 et 42 ). Leur organisation structurée est particulièrement frappante comparée à celle du débitage, très opportuniste, dont les vestiges, notamment des nucléus peu prédéterminés et des " éclats aussi grands, et peutêtre aussi minces que possible ", sont dispersés dans tout le campement (Pigeot 2003, p. 129). On observe donc à Ponsonby des comportements différenciés entre le débitage, effectué de manière conjoncturelle en fonction des besoins, et le façonnage " réalisé par une seule personne, fabriquant en une seule fois, de bout en bout, la pointe bifaciale désirée " (ibid., p. 157). La fabrication des pointes semble ainsi correspondre à une activité bien localisée dans le temps et dans l'espace, très probablement réservée à une ou deux personnes ayant acquis cette compétence technique.

Si l'on tente une comparaison avec les traditions de production lithique des Indiens canoeros anciens, le seul exemple disponible, celui de Bahia Colorada, témoigne d'une organisation beaucoup plus lâche. Dans ce site, opérations de façonnage et de débitage se mêlent, réalisées de manière opportuniste au fur et à mesure des besoins. L'ensemble des vestiges est ainsi très dispersé malgré quelques petits regroupements : concentration d'armes et d'outils brûlés dans une zone sans doute rituelle, plus forte représentation d'outils dans l'habitation, petit tas d'éclats évacués pêle-mêle en périphérie... (Legoupil 1997). Mais ces concentrations sont sans rapport avec la production et aucun atelier de travail spécialisé n'a été clairement identifié.

Ces comportements différenciés n'ont jusqu'à présent été envisagés que sur deux sites, faute d'études techniques et spatiales détaillées. On peut poser l'hypothèse d'une organisation du travail plus spécialisée, plus systématique, et donc d'une organisation sociale plus structurée pour les groupes à grandes pointes que chez les Canoeros anciens. Mais ce point demandera à être vérifié sur d'autres gisements. 
La fonction des pointes

Même si la fonction la plus communément admise pour ces pièces est celle de pointe d'arme (de poignard, sagaie, ou lance), d'autres fonctions sont envisageables comme celle de couteau. En fait, peu d'éléments concrets peuvent nous renseigner sur leur usage. Aucun stigmate tracéologique n'a été observé sur les tranchants des 21 pièces bifaciales du locus 1 de Ponsonby analysées par Christensen (2003), alors que des traces de travail de matières minérales, animales et végétales ont été relevées sur des grattoirs, racloirs et éclats retouchés. Par ailleurs, les cassures des pièces bifaciales retrouvées dans les ateliers semblent dues à des accidents de fabrication, même si quelques fractures en languette peuvent laisser envisager un accident d'utilisation en percussion.

Si l'on examine l'ensemble de la panoplie technique du groupe, on constate que l'apparition des grandes pointes s'accompagne d'une régression du nombre des harpons en os, si représentatifs des Indiens canoeros anciens, ainsi que des couteaux sur éclats retouchés. Les grandes pointes lithiques ont-elles remplacé les pointes de harpons en os ?Étaient-elles mieux adaptées à la chasse aux guanacos qu'il convient de blesser ou de tuer, et non de retenir (fonction principale du harpon pour éviter à l'animal marin de couler) ? Ou bien ont-elles servi de couteaux (d'autant plus efficaces qu'ils étaient denticulés), rendant moins nécessaire le simple usage du fil d'éclats et, pourquoi pas, de couteaux emmanchés comme on en connaît au Néolithique français (Figure 7) ? Mais, dans ce cas, pourquoi toutes les pièces portent-elles une extrémité appointée ? et pourquoi seraient-elles presque toujours symétriques ? Enfin, une dernière hypothèse serait d'envisager un usage en racloir, étant donné la parenté entre certaines pièces bifaciales et les grands racloirs à retouches couvrantes, bien connus à cette

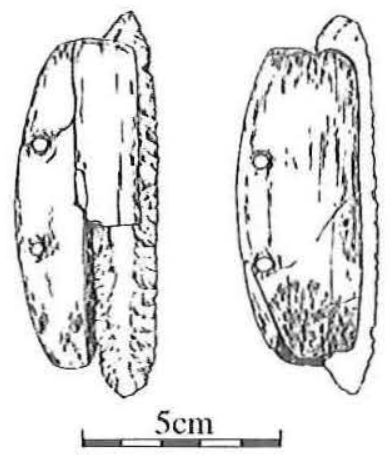

FIG. 7 - Couteau emmanché néolithique, Perte de la Jonquière, Foissac (D'après Camps-Fabrer et Ramseyer 1993, figure 2). 
période. Elle est cependant peu probable compte tenu de la symétrie des pointes et de leur grand allongement, défavorable à une bonne préhension. De plus, la finition denticulée, si efficace pour couper, serait évidemment une aberration en raclage de peau.

En fait, la difficulté d'interprétation de ces pièces pourrait peut-être provenir de leur caractère multifonctionnel : arme et outil.

\section{UN CARACTÈRE SYMBOLIQUE OU SOCIAL ?}

Quel qu'ait été leur usage, il est manifeste que les grandes pointes de Ponsonby ont acquis une valeur ajoutée sans rapport avec leur rôle pratique : une fonction symbolique ou sociale.

En effet, bien des particularités de ces objets sont assez remarquables et témoignent d'un soin attentif à les rendre ainsi. Déjà, on l'a vu, leur module exceptionnel : si long, si mince et si allongé, alors que ces pointes devenaient ainsi fragiles et perdaient en fonctionnalité, particulièrement pour un usage en impact. Par ailleurs, leur réalisation témoigne d'une haute qualité technique : enchâ̂nement programmé des modes de détachement (direct dur, tendre, pression) et fort automatisme des gestes et des objectifs, visible sur de nombreuses pointes. Ces caractères indiquent que des artisans avertis ont dû réaliser ces pièces exceptionnelles à la suite d'un apprentissage privilégiant la conception du modèle le plus long, le plus mince, le plus régulier - on serait tenté de dire le plus esthétique - au détriment de l'économie opératoire (risque de cassures au façonnage) et de l'efficacité de l'objet.

La valeur symbolique de certaines productions matérielles a été fréquemment observée, notamment en ethnologie, plus particulièrement pour les pointes d'armes, attribut hautement viril. Des explications similaires ont parfois été avancées pour des cas archéologiques. Ainsi Cauvin (1994, p. 169) considère que la technologie des pointes lithiques du Néolithique proche oriental (PPNB) représentait « un secteur de prestige appelant un investissement artisanal supplémentaire et une recherche esthétique inexplicables autrement ». Une interprétation similaire a été donnée récemment aux longues feuilles de laurier solutréennes d'Europe, dont la perfection et la dimension ( 25 à $35 \mathrm{~cm}$ ) valaient probablement à leurs auteurs, il y a 20000 ans, une reconnaissance sociale importante, voire une représentation symbolique de capacités hors du commun (voir, par exemple, Aubry et al. 2007). Les grandes pointes n'ont du reste peut-être pas représenté le seul marqueur technique fort de cette culture. On peut entrevoir une recherche d'exploit technique valant reconnaissance sur d'autres pièces, notamment en os de grand cétacé, animal souvent à forte valeur symbolique chez les chasseurscueilleurs maritimes. Ainsi, pour les pointes de sagaies de Ponsonby, massives et parfaitement rectilignes, qui, entières, devaient facilement dépasser $30 \mathrm{~cm}$ de 
longueur (Legoupil 2003d). De même, certaines pointes de harpons se distinguent, par leur longueur, du lot commun des harpons archéologiques, limités à $18-20 \mathrm{~cm}$ avant l'introduction d'outils métalliques modernes. C'est le cas, par exemple, d'un grand harpon très massif de $30 \mathrm{~cm}$ de long découvert en janvier 2008 à Offing (Figure 8, premier à gauche) : il fut non seulement très probablement réalisé sur os mandibulaire de cétacé, mais en outre décoré, ce qui est rare dans les sites à grandes pointes. On pourrait citer d'autres pièces comme ce harpon de $25 \mathrm{~cm}$, récemment découvert dans un site daté d'environ 5000 ans, à Bahia Valentin 11 (BV 11), dans le canal Beagle (Zangrando et al. s. d.) et qui porte des reliefs en bouton dont l'un au moins pourrait avoir une valeur décorative (Figure 8, deuxième à gauche).

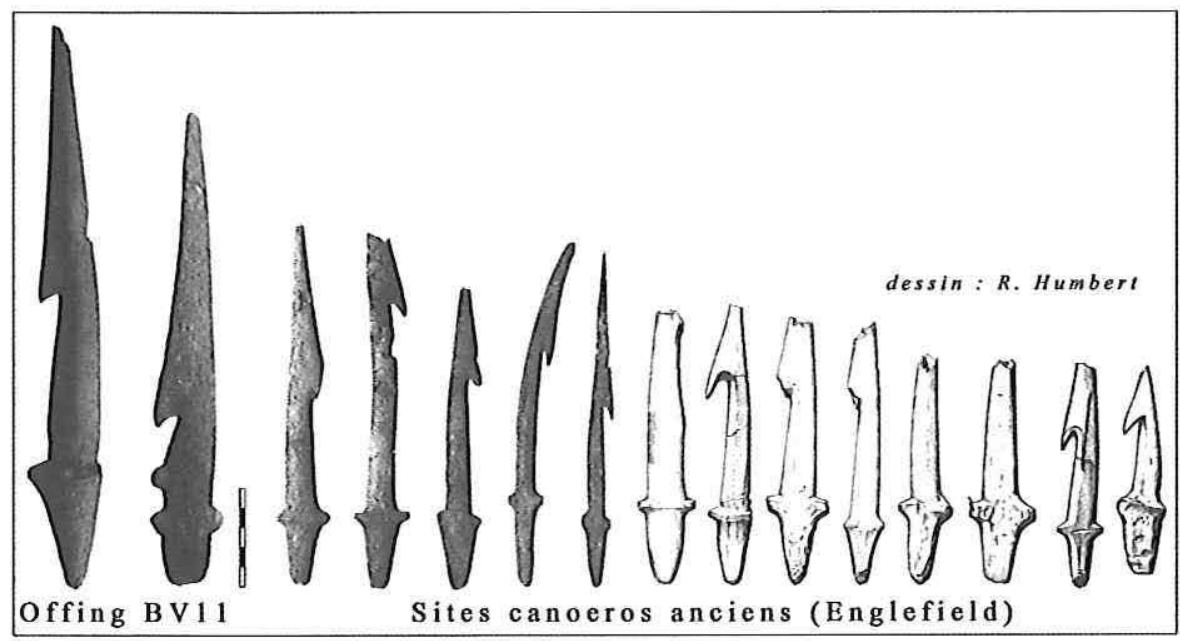

FIG. 8 - Grands harpons des sites anciens et des sites d'Offing (premier à gauche) et de Cabo Valentin 11 (deuxièmeà gauche).

\section{CONCLUSION}

Les grandes pointes bifaciales foliacées sont apparues en Patagonie dans un contexte culturel dont la stabilité technique, économique et, sans doute, sociale a souvent été soulignée, notamment pour les groupes maritimes, au cours de la seconde moitié de l'Holocène (Legoupil 1992; Orquera et Piana 1999). La panoplie lithique et osseuse de ces chasseurs-cueilleurs est globalement la même durant des millénaires (des pointes d'armes, des grattoirs, des racloirs, des poinçons...). Les principaux mécanismes cognitifs techniques sont en place : la taille en percussion dure, en percussion tendre, la pression, le façonnage bifacial, 
l'exploitation des rognons selon une technique discoïde ou proche de la famille Levallois, un débitage laminaire (rare mais connu), etc.

Dans ce contexte technique relativement homogène, les grandes pointes présentent les caractères typiques d'une véritable tradition culturelle, révélés non seulement par les particularités de la structure opératoire de la chaîne de façonnage, mais aussi par l'analyse des objets eux-mêmes. Mais, pour atteindre ces traits diagnostiques, en rester au premier niveau d'analyse aurait été insuffisant, selon l'interprétation classique des degrés du fait de Leroi-Gourhan ${ }^{3}$. Ainsi la simple définition des grandes pointes foliacées ne suffit pas. On a vu que les pointes de Ponsonby, Lancha Packewaïa et de la dizaine de sites attribués à ce groupe ont permis d'identifier des critères diagnostiques personnalisés et donc culturellement pertinents (automatisme technique, standard morphologique, soin d'exécution, probable valeur sociale...). Il existe donc bien un type de pointe représentatif d'un faciès culturel que, par facilité, on serait tenté de nommer du nom du site éponyme, le premier découvert : Ponsonby.

Resterait à comprendre l'origine de ces pointes et le contexte culturel de leur apparition : emprunt? invention ? Rappelons les facteurs qui pourraient expliquer cette innovation : la perte de l'obsidienne, par exemple, matériau si utilisé auparavant dans la mer d'Otway et la partie centrale du détroit de Magellan et qui disparaît vers cette période, pour reparaître ensuite (San Roman et Prieto 2004) ; mais aussi le choix d'une nouvelle stratégie de chasse entraînant peut-être le besoin d'un nouvel équipement; ou encore un événement historique comme l'arrivée d'une nouvelle population ou l'emprunt d'une nouvelle idée...

Ainsi, la disparition de l'obsidienne (quelle qu'en soit la cause) aurait pu entrainner le choix, par défaut, de la lutite et de la rhyolithe et, au-delà, l'émergence d'une nouvelle technologie, alternative, destinée aux armes de chasse et outils de boucherie. De même, l'importance prise par la chasse aux guanacos par rapport à l'exploitation des ressources marines, et notamment des pinnipèdes (sauf à Offing), pourrait également expliquer la relative pauvreté des harpons et le spectaculaire développement des pointes. Les besoins ne sont pas les mêmes dans les deux cas : un chasseur de guanaco doit avant tout tuer ou blesser sa proie qu'il traquera et poursuivra à cet effet. En revanche, un chasseur de pinnipède ou de dauphin doit, non seulement tuer, mais aussi retenir l'animal pour éviter qu'il ne coule, d'où l'importance fondamentale de la ligne qui fait que le harpon s'apparente autant à un hameçon qu'à une arme de jet. Si l'utilisation des grandes pointes est liée à l'exploitation des guanacos, la question qui se pose est de savoir pourquoi une population, à l'origine maritime, a adopté une économie de subsistance terrestre. Raison climatique ? Événement biologique ? Compétition territoriale ? Mais, si elle est d'origine terrestre, pourquoi ses techniques de chasse ne sont-elles pas identiques à celles des chasseurs de guanacos de la pampa continentale ou de Terre de Feu qui, rappelons-le, n'ont jamais fabriqué de grandes pointes de ce type ? 
Enfin, il reste à s'interroger sur l'origine géographique de cette technologie. De grandes pointes bifaciales similaires, mais légèrement plus petites, sont apparues, depuis quelques années, au nord des archipels, dans la partie septentionale des îles Guaitecas et dans la région de Puerto Montt-Chiloé. Les sites d'où elles proviennent, Piedra Azul (Gaete et al. 2004), Puente Quilo (Rivas et al. 1999; Ocampo et Rivas 2004) et Guaitecas 10 (Porter 1993), présentent des datations qui s'étalent entre 4000 et $5500 \mathrm{BP}$, ce qui les situe globalement dans le cadre chronologique de la culture à grandes pointes de la zone australe. En attendant que des recherches dans la partie centrale des archipels puissent éclairer les relations entre ces deux régions distantes de près de $1000 \mathrm{~km}$, seules de nouvelles analyses, tant sur le plan chronostratigraphique que morphologique et technique, pourraient permettre de mesurer le degré de parenté de ces deux séries et de juger de leur ancienneté relative. Encore faut-il se placer au bon niveau d'analyse : soit par l'observation des traits culturels diagnostiques révélés par les objets finis, soit par celle des chaînes opératoires de façonnage, ce qui implique une vision chrono-spatiale de l'ensemble des vestiges (objets finis, nucléus et déchets de fabrication) permettant une reconstitution qui va de l'acquisition de la matière première jusqu'à l'abandon des produits après consommation.

Pour résoudre le dilemme entre invention (ou plutôt innovation) et emprunt, il faudra donc mettre au jour une documentation archéologique de bonne qualité et des sites qui puissent tenir le rôle de jalons entre ces deux pôles. En attendant, on peut émettre l'hypothèse que les relations entre Puerto Montt-Chiloé et la zone australe étaient moins distendues qu'il n'y paraît et les frontières plus poreuses qu'on ne l'imagine généralement au vu de la grande distance et des difficultés de navigation dans cette région. La cristallisation du modèle des grandes pointes foliacées a pu se produire sans doute très facilement, le terrain étant favorable à l'émergence de ces objets spectaculaires. Depuis Leroi-Gourhan (1973, p. 394), on sait que l'on n'emprunte que ce qu'on était prêt à inventer : « [...] l'emprunt pur n'est possible que pour un groupe dont le milieu technique possède déjà le moyen de le recevoir [...]. Pour l'invention, la même condition s'impose : le groupe n'invente que s'il est en possession d'éléments préexistants suffisants pour fonder l'innovation. Une certaine identité se révèle par conséquent entre l'invention et l'emprunt [...]. Dans nombre de cas, il y a fusion entre l'emprunt et l'invention ".

* Manuscrit reçu en septembre 2008, accepté pour publication en septembre 2009.

\section{Notes}

1. Indios canoeros ( Indiens en canot ») est le nom communément adopté aujourd'hui pour les nomades marins des archipels de Patagonie et de Terre de Feu. Il concerne l'ensemble des ethnies (Chono, Alakaluf et Yamana) reconnues par l'ethnologie classique dont la division est aujourd'hui parfois contestée. 
2. Pour le concept de "chaîne opératoire » de Leroi-Gourhan (1965) et de l'École française d'ethnologie, voir les historiques récents de Schlanger (2004) et Pigeot (s. d.).

3. «Les faits présentent des degrés de valeur différente et ce ne sont pas les caractères du premier degré, généralement liés à la tendance, qui sont les plus intéressants, mais ceux du second ou du troisième degré, proprement attachés au peuple ou au groupe de peuple dont le fait étudié est issu » (Leroi-Gourhan 1971, p. 30).

\section{RÉFÉRENCES CITÉES}

Álvarez Myrian

2007 « Procesos de producción y uso de instrumentos bifaciales entre los grupos canoeros del canal Beagle ", in Flavia Morello, Mateo Martinic, Alfredo Prieto et Gabriel Bahamonde (éds), Arqueología de Fuego-Patagonia. Levantando piedras, desenterrando huesos... y develando arcanos, Ediciones CEQUA, Chile, pp. 247-255.

Aubry Thierry, Miguel Alameida, Javier Mangando Llach, Maria João Neves, JeanBaptiste Peyrouse et Bertrand WaLter

2007 "Une énigme préhistorique : les grandes feuilles de laurier solutréennes 》, Archéologia, 444, pp. 36-44.

BIRD Junius

1993 Viajes y arqueología en Chile Austral, Ed. Univ. de Magallanes, Punta Arenas (Chili) [1988 pour l'édition anglaise].

CAMPS-FABrer Henriette et Denis RamSEyer

1993 "Fiche manches en os rainurés latéralement», Fiches typologiques de l'industrie osseuse préhistorique, Édition CEDARC, cahier VI, Treignes (Belgique).

Cauvin Jean

$1994 \quad$ Naissances des divinités, naissance de l'agriculture : la révolution des symboles au Néolithique, CNRS éditions, Paris.

Clapperton Chalmers M.

1992 «La última glaciación y deglaciación en el Estrecho de Magallanes : implicaciones para el poblamiento de Tierra del Fuego ", Anales del Instituto de la Patagonia, Punta Arenas (Chili), 21, pp. 113-128.

Christensen Marianne

2003 " Analyse fonctionnelle des outils de pierre taillée », in Dominique Legoupil (éd.), Les chasseurs-cueilleurs de Ponsonby (Patagonie australe) et leur environnement du VI $T^{e}$ au $I I T^{e}$ millénaire $B C$, Univ. de Magallanes, Punta Arenas (Chili), pp. 203-213.

EMPERAire José

1988 " Paisajes y hombres prehistóricos de la Patagonia », Anales del Instituto de la Patagonia, 18, pp. 79-94 [1953]. 
Emperaire José et Annette Laming

1961 «Les gisements des îles Englefield et Vivian dans la mer d'Otway (Patagonie australe) ", Journal de la Société des Américanistes, 50, pp. 7-75.

Gaete Nelson, Ximena Navarro, Florence Constantinescu, Carlos Mera, Daniel Selles, María-Eugenia Solari, María Loreto Vargas, Doris Oliva et Luis Durán

2004 "Una mirada al modo de vida canoero del mar interior desde Piedra Azul », Chungara. Revista de Antropología Chilena, numéro spécial, 1, pp. 333-346.

LAMING-EMPERAire Annette

1967-1968 «Cadre chronologique provisoire de la Préhistoire de Patagonie et de Terre de Feu chiliennes ", Boletín del Museo nacional de Historia natural, XXX, pp. 221-236.

LeFÈvre Christine, Sébastien LePETZ et Dominique LegoupIL

2003 "Chasseurs terrestres, chasseurs maritimes, exploitation des ressources animales dans le locus 1 », in Dominique Legoupil (éd.), Les chasseurscueilleurs de Ponsonby (Patagonie australe) et leur environnement du VI au $I I I^{e}$ millénaire $B C$, Univ. de Magallanes, Punta Arenas (Chili), pp. 63-116.

LegoupIL Dominique

1988 "Últimas consideraciones sobre la dataciones del sitio de isla Englefield (Seno Otway) ", Anales del Instituto de la Patagonia. Serie ciencias humanas, Punta Arenas (Chili), 18, pp. 86-87.

1992 "Une méthode d'interprétation ethnoarchéologique en deux temps : un modèle culturel, sur 6000 ans, chez les nomades marins de Patagonie ", Actes des $\mathrm{XII}^{\mathrm{e}}$ Rencontres internationales d'archéologie et d'histoire d'Antibes, Ethnologie: justification, problèmes et limites, ADCPA, Juan-les-Pins, pp. 357-375.

1992-1993 Ponsonby et la prospection de la mer de Skyring sud, rapport de mission pour le ministère des Affaires étrangères, Paris [manuscrit].

1997 Bahia Colorada : les premiers chasseurs de mammiferes marins de Patagonie australe, ADPF, coll. " Recherches sur les Civilisations », Paris.

2003a "Le site archéologique», in Dominique Legoupil (éd.), Les chasseurscueilleurs de Ponsonby (Patagonie australe) et leur environnement du VI au III millénaire $B C$, Univ. de Magallanes, Punta Arenas (Chili), pp. 19-62.

2003b "L'utilisation de la pierre naturelle, polie et piquetée », in Dominique Legoupil (éd.), Les chasseurs-cueilleurs de Ponsonby (Patagonie australe) et leur environnement du VI $I^{2}$ au III millénaire BC, Univ. de Magallanes, Punta Arenas (Chili), pp. 215-224.

2003c "La industria en hueso ", in Dominique Legoupil (éd.), Les chasseurscueilleurs de Ponsonby (Patagonie australe) et leur environnement du VI au III millénaire BC, Univ. de Magallanes, Punta Arenas (Chili), pp. $225-262$. 
2003d «L'organisation sociale des campements », in Dominique Legoupil (éd.), Les chasseurs-cueilleurs de Ponsonby (Patagonie australe) et leur environnement du $V I^{e}$ au $I I T^{e}$ millénaire $B C$, Univ. de Magallanes, Punta Arenas (Chili), pp. 263-300.

LegouplL Dominique et Michel Fontugne

1997 «El poblamiento maritimo en los archipielagos de Patagonia: nucleos antiguos y dispersion reciente ", Anales del Instituto de la Patagonia. Serie ciencias humanas, 25, Punta Arenas (Chili), pp. 75-87.

Legoupil Dominique, Marianne Christensen, Mathieu Langlais, Sébastien Lepetz et Kai SALAS

2003 Les voies de peuplement des archipels de Patagonie, région d'Ultima Esperanza et de Chiloé, rapport de mission pour le ministère des Affaires étrangères, Paris.

Legoupll Dominique, Philippe Bearez, Marianne Christensen, Christine Lefèvre, Flavia Morello, Kai Salas et Manuel San Roman

2007 Le faciès culturel des populations à grandes pointes en Patagonie : les sites de l'île Dawson, rapport de mission pour le ministère des Affaires étrangères, Paris.

Lepetz Sébastien, Christine Lefèvre et Éric Pellé

2003 "Les guanacos du locus 2: note sur un dépôt naturel », in Dominique Legoupil (éd.), Les chasseurs-cueilleurs de Ponsonby (Patagonie australe) et leur environnement du $V T^{e}$ au III millénaire $B C$, Univ. de Magallanes, Punta Arenas (Chili), pp. 415-418.

LEROI-GOURHAN André

1965 Le Geste et la Parole. 2. La mémoire et les rythmes, Albin Michel, Paris.

1971 Évolution et technique. 2. Milieu et techniques, Albin Michel, Paris [1945].

Miranda P.

1967 Diario de las excavaciones realizadas en Puerto del Hambre, Instituto de la Patagonia, Punta Arenas (Chili).

Morello Flavia, Manuel San Román, Alfredo Prieto et Charles Stern

2002 "Puntas de proyectil lanceoladas en Patagonia », Anales del Instituto de la Patagonia, 30, pp. 155-166.

Morello Flavia, Manuel San Román et Alfredo Prieto

2004 "Obsidiana verde en Fuego-Patagonia : distribución y estrategias tecnológicas ", in María Teresa Civalero, Pablo Marcelo Fernández et Ana Gabriela Guráieb (éds), Contra viento y marea. Arqueología de Patagonia, INAP/Sociedad Argentina de Antropología, Buenos Aires, pp. 149-165.

Ocampo Carlos et Pilar Rivas

2004 "Poblamiento temprano de los extremos geográficos de los canales patagónicos: Chiloé e isla Navarino 1 ", Chungara. Revista de Antropología Chilena, numéro spécial, 1, pp. 317-331. 
Orquera Luis Abel, Arturo Emilio Sala, Ernesto Luis Piana et Alicia Haydée Tapia

1977 Lancha Packewaïa, Ed. Huemul, Buenos Aires.

Orquera Luis Abel et Ernesto Luis Piana

1986-1987 «Composición tipológica y datos tecnomorfológicos y tecnofuncionales de los distintos conjuntos arqueológicos del sitio Túnel I (Tierra del Fuego, Rep. Argentina) ", Relaciones de la Sociedad Argentina de Antropología, XVII (1), pp. 201-239.

1993-1994 "Lancha Packewaïa : actualización y rectificaciones », Relaciones de la Sociedad Argentina de Antropologia, XIX, pp. 325-362.

1999 Arqueología de la región del canal Beagle, Sociedad Argentina de Antropología, Buenos Aires.

\section{OrTIZ-Troncoso Omar R.}

1973 "Aspectos arqueológicos de la Península de Brunswick », Anales del Instituto de la Patagonia, Punta Arenas (Chili), IV (1-3), pp. 109-129.

1979 "Punta Santa Ana et Bahia Buena : deux gisements sur une ancienne ligne de rivage dans le détroit de Magellan », Journal de la Société des Américanistes, 66, pp. 133-204.

1980-1981 «Inventory of radiocarbon dates from Southern, Patagonia and Tierra del Fuego », Journal de la Société des Américanistes, LXVII, pp. 185-212.

Pigeot Nicole

2003 «L'économie de la taille de la pierre », in Dominique Legoupil (éd.), Les chasseurs-cueilleurs de Ponsonby (Patagonie australe) et leur environnement du $V I^{e}$ au $I I I^{e}$ millénaire $B C$, Univ. de Magallanes, Punta Arenas (Chili), pp. 115-164.

s. d. "Chaînes opératoires : contexte théorique et potentiel cognitif », in René Treuil (éd.), Archéologie cognitive, Maison des Sciences de l'Homme/ Ophrys, coll. « Cognitique », Paris [sous presse].

Pigeot Nicole et Valérie SchidlowsKy

1997 "L’équipement en pierre taillée », in Dominique Legoupil (éd.), Bahia Colorada: les premiers chasseurs de mammifères marins de Patagonie australe, ADPF, coll. « Recherches sur les Civilisations », Paris.

PORTER Charles

1993 "GUA-010, un sitio costero erosionado en una zona sísmica activa », Actas del XII Congreso Nacional de Arqueología Chilena, I, Simposios, Boletín del Museo Regional de la Araucanía, tome 1, pp. 81-88.

Rivas Pilar, Carlos Ocampo et Eugenio Aspillaga

1999 «Poblamiento temprano de los canales patagónicos : el núcleo ecotonal septentrional », Anales del Instituto de la Patagonia, Punta Arenas (Chili), 27, pp. 221-230.

SAN Roman Manuel

2005 "Nuevos hallazgos de sitios de cazadores recolectores marinos tempranos en isla Englefield, mar de Otway », Magallania, 33 (2), pp. 173-176. 


\section{San Roman Manuel et Alfredo Prieto}

2004 "(Dis)continuidad del uso de obsidiana verde entre poblaciones de adaptación marítima del mar de Otway y estrecho de Magallanes ", in María Teresa Civalero, Pablo Marcelo Fernández et Ana Gabriela Guráieb (éds), Contra viento y marea. Arqueología de Patagonia, INAP/Sociedad Argentina de Antropología, Buenos Aires, pp. 571-580.

SCHLANGER Nathan

2004 " "Suivre les gestes, éclat par éclat". La chaîne opératoire d'André LeroiGourhan ", in Françoise Audouze et Nathan Schlanger (éds), Autour de l'homme. Contexte et actualité d'André Leroi-Gourhan, APDCA, Antibes, pp. 127-148.

SchidLowsKy Valérie

2001 «Les premiers chasseurs maritimes et les chasseurs terrestres de Patagonie australe : comportements techno-économiques et identité culturelle. Contribution de la technologie lithique », BAR International, 954, coll. « Monographs in American Archaeology » 10, Paris.

2003 «Les outils en pierre taillée : singularité des concepts et influences régionales ", in Dominique Legoupil (éd.), Les chasseurs-cueilleurs de Ponsonby (Patagonie australe) et leur environnement du VI au III millénaire BC, Univ. de Magallanes, Punta Arenas (Chili), pp. 165-204.

2006 «Identité culturelle et technologie lithique: les comportements technoéconomiques des premiers chasseurs maritimes et des chasseurs terrestres de Patagonie australe », in Hugo Daniel Yacobaccio et D. E. Olivera (éds), Change in the Andes: origins of social complexity, pastoralism and agriculture, BAR international, 1524, Oxford, pp. 191-201 [2001].

Stern Charles R. et Alfredo Prieto

1991 "Obsidiana verde de los sitios arqueólogicos en los alrededores del mar de Otway, Magallanes (Chile) », Anales del Instituto de la Patagonia, 20, pp. 139-144.

Terradas Xavier, Felicia Plana et José S. Chinchón

1991 "Aplicación de técnicas analíticas al estudio de materias primas líticas prehistóricas ", Arqueología, Madrid, pp. 141-167.

Terradas Xavier

2003 "Caracterización de las materias primas líticas », in Dominique Legoupil (éd.), Les chasseurs-cueilleurs de Ponsonby (Patagonie australe) et leur environnement du VI $I^{e}$ au $I I^{e}$ millénaire $B C$, Univ. de Magallanes, Punta Arenas (Chili), pp. 419-423.

ZangRando Francesco, Augusto Tessone et Martín Vásquez

s. d. «El uso de espacios marginales del archipiélago fueguino : implicaciones de la evidencia arqueológica de Bahia Valentin ", Cazadores-recolectores y pescadores del litoral sudamericano sudoccidental, VII Jornadas de Arqueología de Patagonia, Ushuaia, 21-25 de abril 2008. 